\title{
Semiregular Modules with Respect to a Fully Invariant Submodule ${ }^{\#}$
}

\author{
Mustafa Alkan ${ }^{1, *}$ and A. Çiğgdem Özcan ${ }^{2}$ \\ ${ }^{1}$ Department of Mathematics, Akdeniz University, \\ Antalya, Turkey \\ ${ }^{2}$ Department of Mathematics, Hacettepe University, \\ Beytepe, Ankara, Turkey
}

\begin{abstract}
Let $M$ be a left $R$-module and $F$ a submodule of $M$ for any ring $R$. We call $M$ $F$-semiregular if for every $x \in M$, there exists a decomposition $M=A \oplus B$ such that $A$ is projective, $A \leq R x$ and $R x \cap B \leq F$. This definition extends several notions in the literature. We investigate some equivalent conditions to $F$-semiregular modules and consider some certain fully invariant submodules such as $Z(M), \operatorname{Soc}(M), \delta(M)$. We prove, among others, that if $M$ is a finitely generated projective module, then $M$ is quasi-injective if and only if $M$ is $Z(M)$-semiregular and $M \oplus M$ is CS. If $M$ is projective $\operatorname{Soc}(M)$-semiregular module, then $M$ is semiregular. We also characterize QF-rings $R$ with $J(R)^{2}=0$.
\end{abstract}

Key Words: Semiregular modules; CS modules; Quasi-injective modules; ACS rings; QF rings.

Mathematics Subject Classification: 16D40;16D50;16L60.

${ }^{\#}$ Communicated by R. Wisbauer

*Correspondence: Mustafa Alkan, Department of Mathematics, Akdeniz University, Antalya 07058, Turkey; E-mail: alkan@akdeniz.edu.tr. 


\section{INTRODUCTION}

Perfect, semiperfect and semiregular (or $f$-semiperfect) rings constiture the class of rings that posses beautiful homological and non homological properties. The concept of semiperfect rings has been generalized to semiperfect modules by Mares (1963). Mares calls a module $M$ a semiperfect module if every quotient of $M$ has a projective cover. Nicholson (1976) proves that a projective module $M$ is semiperfect if and only if it is semiregular, $\operatorname{Rad}(M) \ll M$ and $M / \operatorname{Rad}(M)$ is semisimple. Semiregular modules are known as a unified generalization of semiperfect modules and regular modules of Zelmanowitz. There has been a great deal of work on semiregular modules by several authors (e.g., Azumaya, 1991; Nicholson, 1976; Wisbauer, 1991; Xue, 1995).

Zhou (2000) defines $\delta$-semiregular and $\delta$-semiperfect rings as a generalization of semiregular and semiperfect rings. On the other hand, Nicholson and Yousif (2001) consider $I$-semiregular rings for an ideal $I$ of a ring $R$ and study $Z\left({ }_{R} R\right)$-semiregular rings. Now in this paper, we define $F$-semiregular modules $M$ for a submodule $F$ of a module $M$ and consider some certain fully invariant submodules such as $Z(M)$, $\operatorname{Soc}(M), \delta(M)$ (is defined in Zhou, 2000).

If $M$ is semiregular, then for every $x \in M$ there exists a decomposition $M=$ $A \oplus B$ such that $A \leq R x$ is projective and $B \cap R x \ll M$ or equivalently $B \cap R x \leq$ $\operatorname{Rad}(M)$. Therefore, here we may consider any (fully invariant) submodule $F$ or $M$ instead of $\operatorname{Rad}(M)$, and we denote such modules as $F$-semiregular modules. In Sec. 2, we investigate the equivalent conditions to $F$-semiregular modules inspired by Nicholson and Yousif's results. Some of their results are directly generalized but some are not, and we define $\left(S_{1}\right)$ and $\left(S_{2}\right)$ properties for them.

In Sec. 3, we consider $Z(\cdot)$-semiregular modules. We prove that for a finitely generated projective module $M, M$ is quasi-injective if and only if $M$ is $Z(M)$-semiregular and $M \oplus M$ is CS.

In the last section, we consider $\operatorname{Soc}(\cdot)$-semiregular and $\delta(\cdot)$-semiregular modules and investigate the relationship between them. We prove that if $M$ is a countably generated $\delta(M)$-semiregular module with $\delta(M) \ll_{\delta} M$ then $M$ is isomorphic to a direct sum of projective cyclic submodules of $M$. Any projective $\operatorname{Soc}(M)$ semiregular module $M$ is semiregular. And we characterize left Artinian rings $R$ with $J(R)^{2}=0$ and quasi-Frobenius (QF) rings $R$ with $J(R)^{2}=0$. At the end of the paper, we give some counter examples.

Throughout this paper, $R$ denotes an associative ring with identity and modules $M$ are unitary left $R$-modules. For a module $M, \operatorname{Rad}(M)$ and $Z(M)$ are the Jacobson radical and the singular submodule of $M$. We write $J(R)$ for the Jacobson radical of $R$. The dual of $M$ is denoted by $M^{*}=\operatorname{Hom}_{R}(M, R)$. A submodule $N$ of $M$ is called small in $M$, denoted by $N \ll M$, whenever for any submodule $L$ of $M, N+L=M$ implies $L=M$. Dually we use $N \leq_{e} M$ to signify that $N$ is an essential submodule of $M$. For a direct summand $K$ of $M$ we write $K \leq{ }^{\oplus} M$.

A submodule $N$ of a module $M$ is said to lie over a summand of $M$ if there exists a decomposition $M=A \oplus B$ such that $A \leq N$ and $B \cap N$ is small in $M$. An element $x$ in $M$ is called regular if $(x \alpha) x=x$ for some $\alpha \in M^{*}$. Zelmanowitz (1973) calls a module regular if each of its elements is regular, equivalently if every finitely generated submodule is a projective summand. Nicholson (1976) calls an element 
$x$ and $M$ semiregular if $R x$ lies over a projective summand of $M$. A module called semiregular if each of its elements is semiregular.

\section{F-SEMIREGULAR MODULES}

In this chapter, we investigate some equivalent conditions to $F$-semiregular modules.

Definition 2.1. Let $F$ be a submodule of an $R$-module $M$. An element $x$ in $M$ is said to be $F$-semiregular in $M$ if there exists a decomposition $M=A \oplus B$ such that $A$ is projective, $A \leq R x$ and $R x \cap B \leq F$. A module $M$ is called an $F$-semiregular module if every elements $x$ in $M$ is $F$-semiregular.

Clearly the class of $F$-semiregular modules contains all regular modules. Also $M$ is semiregular if and only if $M$ is $\operatorname{Rad}(M)$-semiregular. If $M$ is semiregular and $F$ is a submodule of $M$ such that $\operatorname{Rad}(M) \leq F$ then $M$ is $F$-semiregular. For $M=R$ and an ideal $F=I, I$-semiregularity of rings is defined by Nicholson and Yousif (2001). Now we consider the module theoretic version of some results of Nicholson and Yousif.

Proposition 2.2. Let $F$ be a submodule of a module $M$. Then the following conditions are equivalent for $x \in M$.

(1) $x$ is F-semiregular.

(2) There exists $\alpha \in M^{*}$ such that $(x \alpha)^{2}=x \alpha$ and $x-(x \alpha) x \in F$.

(3) There exists a homomorphism $\gamma$ from $M$ to $R x$ such that $\gamma^{2}=\gamma, M \gamma$ is projective and $x-x \gamma \in F$.

When these conditions hold we have

(4) There exists a regular element $y \in R x$ such that $x-y \in F$ and $R x=$ $R y(x-y)$. If $F$ is fully invariant then (1)-(3) are equivalent to (4).

Proof. (1) $\Rightarrow(2)$. Suppose for $x$ in $M$ there exists a decomposition $M=A \oplus B$ such that $A$ is projective, $A \leq R x$ and $R x \cap B \leq F$. Then there exist $x_{i} \in A$ and $\alpha_{i} \in A^{*}=\operatorname{Hom}_{R}(A, R)(i=1, \ldots, n)$ such that $y=\sum_{i=1}^{n}\left(y \alpha_{i}\right) x_{i}$ for any $y \in A$. Hence $\alpha_{i}$ extends to $M$ by $(a+b) \beta_{i}=a \alpha_{i}$. Write $x_{i}=r_{i} x$ with $r_{i} \in R$ and let $\alpha=\sum \beta_{i} r_{i}$. Then $\alpha \in M^{*}$. Write $x=a+b$ with $a \in A, b \in B$. We get $(x \alpha) x=\sum\left(x \beta_{i}\right) r_{i} x=$ $\sum\left(a \alpha_{i}\right) x_{i}=a$. Therefore, $x-a=x-(x \alpha) x=b \in R x \cap B \leq F$.

$(2) \Rightarrow(3)$. Let $x$ and $\alpha$ be as in (2) and let $y=(x \alpha) x$. Then $y=(y \alpha) y$. By Nicholson (1976, Lemma 1.1), $R y$ is a projective submodule of $R x$ and $M=R y \oplus W$ where $W=\{w \in M:(w \alpha) y=0\}$. Let $\gamma: M \rightarrow R y$ be the projection map. Hence it is sufficient to show that $x-x \gamma \in F$. Write $x=r y+w \in M$ where $r \in R$ and $w \in W$. Then $0=(x-r y) \alpha y=(x \alpha) y-r(y \alpha) y=(x \alpha) y-r y$, so $x \gamma=$ $r y=(x \alpha) y=y$. Therefore, $x-x \gamma=x-y \in F$.

$(3) \Rightarrow(1)$. Suppose (3) holds. Then $M=M \gamma \oplus M(1-\gamma)$ and $\operatorname{Rx} \cap M(1-\gamma)=$ $R x(1-\gamma) \leq F$. 
$(2) \Rightarrow(4)$. Let $x, \alpha, y$ and $W$ be as in (2) $\Rightarrow(3)$. Then $W \cap R x=R(x-y)$. Therefore, $R x=R y \oplus R(x-y)$.

(4) $\Rightarrow(1)$. Assume $F$ is fully invariant. Let $x$ and $y$ be as in (4) and let $\alpha \in M^{*}$ be such that $(y \alpha) y=y$. Then $M=R y \oplus W$ where $W=\{w \in M:(w \alpha) y=0\}$. Hence, $R x=R y \oplus(R x \cap W)$. Let $\pi: M \rightarrow W$ be the projection map. Then $R x \cap W=$ $(R x \cap W) \pi=(R x) \pi=(R(x-y)) \pi \leq(F) \pi \leq F$. This completes the proof.

Taking $M=R$ and $F=I$ an ideal of $R$ yields (Nicholson and Yousif, 2001, Lemma 1.1). Our next results gives the characterization of $F$-semiregular modules.

Theorem 2.3. Let $F$ be a fully invariant submodule of a module $M$. Then the following conditions are equivalent.

(1) $M$ is F-semiregular.

(2) For any finitely generated submodule $N$ of $M$, there exists a homomorphism $\gamma$ from $M$ to $N$ such that $\gamma^{2}=\gamma, M \gamma$ is projective and $N(1-\gamma) \leq F$.

(3) For any finitely generated submodule $N$ of $M$, there exists a decomposition $M=A \oplus B$ such that $A$ is a projective submodule of $N$ and $N \cap B \leq F$.

(4) For any finitely generated submodule $N$ of $M, N$ can be written as $N=A \oplus S$ where $A$ is a projective summand of $M$ and $S \leq F$.

When these conditions hold we have

(5) For all $x \in M$, there exists a regular element $y \in M$ such that $x-y \in F$.

(6) Every submodule of $M$ that is not contained in $F$ contains a regular element not in $F$.

(7) $\operatorname{Rad}(M) \leq F$ and $Z(M) \leq F$.

Proof. $\quad(1) \Rightarrow(2)$. Let $N$ be a finitely generated submodule with generators $x_{0}, \ldots, x_{n}$. We use the induction on the generating set. By assumption choose $\beta: M \rightarrow R x_{n}$ such that $\beta^{2}=\beta, \quad M \beta$ is projective and $\left(x_{n}\right)(1-\beta) \in F$. Set $K=R x_{0}(1-\beta)+\cdots+R x_{n-1}(1-\beta)$ and by induction choose $\alpha: M \rightarrow K$ such that $\alpha^{2}=\alpha, M \alpha$ is projective and $K(1-\alpha) \leq F$. Define $\gamma=\beta+\alpha-\beta \alpha$. Then $\gamma=\gamma^{2}$ and $M \gamma=M \beta \oplus M \alpha$ since $\alpha \beta=0$. Hence $M \gamma$ is projective. It is enough to show that $N(1-\gamma) \leq F$. Since $N=K+R x_{n}$ it follows that $M \gamma=M \beta+M \alpha \leq R x_{n}+K=N$. Take $n=a+r x_{n} \in N$ as $a \in K$ and $r x_{n} \in K$ and $r x_{n} \in R x_{n} .\left(a+r x_{n}\right)(1-\gamma)=$ $\left(a+r x_{n}\right)(1-\beta)(1-\alpha)=\left(a(1-\beta)+r x_{n}(1-\beta)\right)(1-\alpha)=a(1-\alpha)+\left(r x_{n}(1-\beta)\right)$ $(1-\alpha) \in F$. Therefore $N(1-\gamma) \leq F$.

$(2) \Rightarrow(3)$. Let $N$ and $\gamma$ be as in (2). Then $N \cap(M)(1-\gamma)=N(1-\gamma)$. Hence, $M=M \gamma \oplus M(1-\gamma), M \gamma$ is projective and $N \cap(M)(1-\gamma)=N(1-\gamma) \leq F$.

(3) $\Rightarrow(2)$. Let $N$ be a finitely generated submodule of $M$. By (3), $M=A \oplus B$ where $A$ is a projective submodule of $N$ and $N \cap B \leq F$. Then $N=A \oplus(B \cap N)$. Now consider the projection map $\pi: M \rightarrow A$. Let $\gamma=\pi i$ where $i$ is the inclusion map from $A$ to $N$. Then $\gamma^{2}=\gamma, M \gamma=A$ is projective and $N(1-\gamma) \leq F$.

$(3) \Rightarrow(4)$. It is clear. 
(4) $\Rightarrow(1)$. Let $N$ be a cyclic submodule of $M$. Then $N=A \oplus S$ with $A$ a projective summand of $M$ and $S \leq F$. Then $M=A \oplus B$ for some $B$. Let $\pi: M \rightarrow B$ be the projection map. Then $N=A \oplus(N \cap B)$ and $N \cap B=(N) \pi=(S) \pi \leq(F) \pi \leq F$.

$(1) \Rightarrow(5)$ and $(1) \Rightarrow(6)$ are by Proposition 2.2(4).

$(1) \Rightarrow(7)$. Note that every cyclic submodule of $\operatorname{Rad} M$ is small in $M$ and every projective singular module is a zero module, so (7) follows from (6) and (Nicholson, 1976, Lemma 1.1).

Observe that $(2) \Leftrightarrow(3) \Rightarrow(1)$ holds for any submodule $F$ of a module $M$.

Note that if $I$ is an ideal of a ring $R$ then $I M$ is a fully invariant submodule of $M$.

Theorem 1.2 in Nicholson and Yousif (2001) follows from Theorem 2.3 by taking $M=R$ and $F=I M$.

Nicholson and Yousif (2001) give a counter example showing that condition (5) in Theorem 2.3 does not imply $I$-semiregularity by taking $M=R=\mathbb{Z}$ and $I=2 \mathbb{Z}$. In Theorem 2.6, we give the equivalence under some conditions. First we give some definitions.

Zhou (2000) defines that a submodule $N$ of a module $M$ is called $\delta$-small in $M$ if $N+K \neq M$ for any proper submodule $K$ of $M / K$ singular, denoted by $N \ll_{\delta} M$.

Lemma 2.4 (Zhou, 2000, Lemma 1.2). Let $N$ be a submodule of a module M. Then $N \ll_{\delta} M$ if and only if $M=X \oplus Y$ for a projective semisimple submodule $Y$ with $Y \leq N$ whenever $X+N=M$.

Also Zhou introduces the following fully invariant submodule of a module $M$.

$\delta(M)=\bigcap\{N \leq M: M / N$ is singular simple $\}$.

Then $\delta(M)$ is the sum of all $\delta$-small submodules of $M$ by Zhou (2000, Lemma $1.5)$, and hence $\operatorname{Rad}(M) \leq \delta(M)$. If every proper submodule of $M$ is contained in a maximal submodule of $M$, then $\delta(M) \ll_{\delta} M$.

Let $F$ be a submodule of a module $M$. Then $F$ is said to satisfy

$\left(R_{1}\right)$ If for every summand $A$ of $M, A \cap F$ lies over a summand of $M$.

$\left(R_{2}\right)$ If for every regular element $y$ in $M, R y \cap F$ lies over a summand of $M$.

$\left(S_{1}\right)$ If for every summand $N$ of $M$, there exists a decomposition $M=A \oplus B$ such that $A \leq N \cap F$ and $B \cap N \cap F \ll_{\delta} M$.

$\left(S_{2}\right)$ If for every regular element $y$ in $M$, there exists a decomposition $M=A \oplus B$ such that $A \leq R y \cap F$ and $B \cap R y \cap F \ll_{\delta} M$.

Clearly $\left(R_{1}\right) \Rightarrow\left(R_{2}\right)$ and $\left(S_{1}\right) \Rightarrow\left(S_{2}\right)$. For $M=R,\left(R_{1}\right) \Leftrightarrow\left(R_{2}\right)$ and $\left(S_{1}\right) \Leftrightarrow\left(S_{2}\right)$. If $F \leq \delta(M)$ then $R y \cap F \leq R y \cap \delta(M)=\delta(R y) \ll_{\delta} M$ for any regular element $y \in M$. Hence $F$ satisfies $\left(S_{2}\right)$. If $F \ll_{\delta} M$, then $F$ satisfies $\left(S_{1}\right)$. We also have the following diagram.

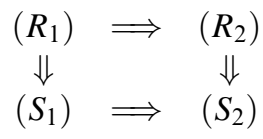

In general $\left(S_{1}\right)$ does not imply $\left(R_{1}\right)$ and $\left(S_{2}\right)$ does not imply $\left(R_{2}\right)$. 
Example 2.5. Let $T$ be the infinite product of $F_{i}$, where each $F_{i}=\mathbb{Z}_{2}$ and let $R$ be the subring of $T$ generated by $\bigoplus_{i \geq 1} F_{i}$ and the identity of $T$. Then $\delta\left({ }_{R} R\right)=$ $\operatorname{Soc}\left({ }_{R} R\right)$ satisfies $\left(S_{1}\right)$ but not $\left(R_{2}\right)$.

Theorem 2.6. Let $F$ be a fully invariant submodule of a module $M$ and satisfy $\left(S_{2}\right)$. Let $x \in M$. If there exists a regular element $y \in M$ such that $x-y \in F$, then $x$ is F-semiregular.

Proof. Let $x \in M$. By assumption there exists a regular element $y \in M$ such that $x-y \in F$ and there is a decomposition $M=K \oplus L$ such that $K \leq F \cap R y$ and $F \cap R y \cap L \ll_{\delta} M$. Since $y$ is regular we have $M=R y \oplus W$ for a submodule $W$ of $M$ and $R y$ is projective. It follows that $M=(R y \cap L) \oplus K \oplus W$ and $F=(R y \cap L \cap F) \oplus K \oplus(W \cap F)$ as $F$ is fully invariant. On the other hand, $F \cap R y \cap L \ll_{\delta} R y+F=R x+F$ as $x-y \in F$ and $R y \leq^{\oplus} M$. Then, by Lemma 2.4, $R x+F=(R x+K+(W \cap F)) \oplus D$ for a projective semisimple submodule $D$ of $F \cap R y \cap L$. Then $R y \cap L=E \oplus D$ where $E=(R y \cap L) \cap(R x+K+(W \cap F))$.

Let $\pi$ be the projection map from $M$ to $E$. Then $E=(R y+F) \pi=(R x+F) \pi=$ $(R x) \pi$. Since $\alpha:=\left.\pi\right|_{R x}$ is an epimorphism and $E$ is projective, $\alpha$ splits. Then there exists $\pi^{\prime}: E \rightarrow R x$ such that $\pi^{\prime} \alpha=1$ and $R x=\operatorname{Im} \pi^{\prime} \oplus \operatorname{Ker}(\alpha)$. Let $A:=\operatorname{Im} \pi^{\prime}$. Since $\operatorname{Ker}(\alpha) \cap A=0$ and $A \leq R x, \operatorname{Ker}(\pi) \cap A=0$. Also $(A) \pi=E$. Hence $\left.\pi\right|_{A}$ is an isomorphism. By Proposition 5.5 in Anderson and Fuller (1974) we have $M=$ $A \oplus D \oplus K \oplus W$ and then $A \cong E$ is projective. On the other hand, $(W+K+D) \cap$ $R x \leq(W+F) \cap(R x+F)=F+(W \cap(R x+F))=F+(W \cap(R y+F))=F+(W \cap$ $(R y+(W \cap F)))=F$. Hence the proof is completed.

Corollary 2.7. Let $F$ be a fully invariant submodule of a module $M$ and satisfy $\left(S_{2}\right)$. Then the following conditions are equivalent.

(1) $M$ is F-semiregular.

(2) For all $x \in M$, there exists a regular element $y \in M$ such that $x-y \in F$.

Corollary 2.8. Let $F$ be a fully invariant submodule of a module $M$ and satisfy $\left(S_{2}\right)$. If $x-y \in F$ and $y$ is $F$-semiregular then $x$ is $F$-semiregular.

Now we give that following lemma without proving because it can be seen by the similar proof of Nicholson (1976, Lemma 1.9).

Lemma 2.9. Let $F$ be a fully invariant submodule of a module $M$. Let $x \in M$. If $\alpha \in M^{*}$ is such that $(x \alpha)^{2}=x \alpha$ and $x-(x \alpha) x$ is F-semiregular, then $x$ is $F$ semiregular.

By the argument in Nicholson (1976, Theorem 1.10) and Corollary 2.8, we have

Theorem 2.10. Let $F$ be a fully invariant submodule of a module $M$ and $M=$ $\bigoplus_{i \in I} M_{i}$ for submodules $M_{i}$. If $M$ is $F$-semiregular then each $M_{i}$ is $F_{i}$-semiregular where $F_{i}=F \cap M_{i}$. The converse is true if $F$ satisfies $\left(S_{2}\right)$. 
Corollary 2.11. Let I be an ideal of a ring $R$ with $I \leq \delta\left({ }_{R} R\right)$. Then $R$ is I-semiregular if and only every projective $R$-module $M$ is IM-semiregular.

Proof. Let $M$ be a projective module. Then $I M \leq \delta(M)$ by Zhou (2000, Lemma 1.9) and so $I M$ satisfies $\left(S_{2}\right)$. Since any projective module is a summand of a free module, the proof is completed by Theorem 2.10 .

Nicholson proves the following theorem in case $F=\operatorname{Rad}(M) \ll M$ in Nicholson (1976, Proposition 1.17). For a submodule $N$ of $M$, if $N \ll_{\delta} M$, then $N$ satisfies $\left(S_{1}\right)$. The converse of this property is not true, for example let $M=\mathbb{Z}\left(p^{\infty}\right)$ be the prüfer p-group. $\operatorname{Rad}(M)=\delta(M)=Z(M)=M$ satisfies $\left(S_{1}\right)$ but not $\delta$-small in $M$. Hence the following theorem generalizes Nicholson (1976, Proposition 1.17).

Theorem 2.12. Let $F$ be a fully invariant submodule of a module M. Consider the following conditions.

(1) $M$ is F-semiregular.

(2) (i) Every finitely generated submodule of $M / F$ is a direct summand.

(ii) If $M / F=A / F \oplus B / F$ where $A / F$ is finitely generated, there exists a decompsition $M=P \oplus Q$ such that $(P+F) / F=A / F$ and $(Q+F) /$ $F=B / F$.

Then $(1) \Rightarrow(2)(\mathrm{i})$. If $M$ is projective, then $(1) \Rightarrow(2)(\mathrm{ii})$. If $M$ is projective and $F$ satisfies $\left(S_{1}\right)$, then $(2) \Rightarrow(1)$.

Proof. $\quad(1) \Rightarrow(2)$. Suppose $M$ is $F$-semiregular and let $A / F \leq M / F$ be finitely generated. Choose a finitely generated submodule $N$ of $M$ such that $A / F=$ $(N+F) / F$. By Theorem 2.3, there is a decomposition $M=C \oplus D$ such that $N=C \oplus(D \cap N)$ and $D \cap N \leq F$. Then $A / F=(N+F) / F=(C+F) / F$. Since $F=(F \cap D) \oplus(F \cap C)$ and $(D+F) \cap(C+F)=(D+(F \cap C)) \cap(C+(F \cap D))=F$, we get $(C+F) / F \oplus(D+F) / F=M / F$. This proves (i).

Now, assume $M / F=A / F \oplus B / F$ where $A / F$ is finitely generated. Choose $N$ and the decomposition of $M$ as above. Then $C+B=M$. Since $C$ is a summand of $M$, apply Nicholson (1976, Lemma 1.16) to write $M=C \oplus Q$ where $Q \leq B$. Then (ii) follows because $(C+F) / F=A / F$ and $(Q+F) / F \leq B / F$.

$(2) \Rightarrow(1)$. Assume that $M$ is projective and $F$ satisfies $\left(S_{1}\right)$. Take a finitely generated submodule $N$ of $M$. By (2), $M / F=(N+F) / F \oplus B / F$ for a submodule $B$ of $M$ with $F \leq B$. Then there exists a decomposition $M=P \oplus Q$ such that $(P+F) / F=(N+F) / F$ and $(Q+F) / F=B / F$. Hence $M=N+Q+F$. Since $F=(P \cap F) \oplus(Q \cap F), M=N+Q+(P \cap F)$. Since $F$ satisfies $\left(S_{1}\right)$, there exists a decomposition $P \cap F=K \oplus S$ where $K$ is a summand of $M$ and $S \ll_{\delta} M$. Then $M=N+Q+K+S=(N+Q+K) \oplus D$ for a submodule $D \leq S$ by Lemma 2.4. Let $T=N+Q+K$ and so $T$ is projective. Since for a submodule $L, K \oplus L=P$ and $M=P \oplus Q=K \oplus L \oplus Q$ we get that $Q \oplus K$ is a summand of $T$. It gives that there is a decomposition $T=(Q \oplus K) \oplus A$ where $A \leq N$ by Nicholson (1976, Lemma 1.16). Since $(Q+K+D) \cap N \leq(Q+F) \cap(N+F)=F, M$ is $F$-semiregular by Theorem 2.3 . 
By the proof of Theorem $2.12(2 \Rightarrow 1)$, we get the following corollary.

Corollary 2.13. Let $F$ be a fully invariant submodule of a module $M$ and satisfy $\left(S_{1}\right)$. If $M$ is $F$-semiregular and $M / F$ is Noetherian, then for any submodule $N$ of $M$ there exists a decomposition $M=A \oplus B$ such that $A \leq N$ and $N \cap B \leq F$.

\section{THE SINGULAR SUBMODULE $Z(M)$}

In this section, we consider the fully invariant submodule $Z(M)$ for a module $M$.

An $R$-module $M$ is called $C S$ (or has $\left(C_{1}\right)$ ), if every closed submodule is a summand. Equivalently, $M$ is $C S$ if and only if every submodule is essential in a summand of $M$. An $R$-module $M$ has $\left(C_{2}\right)$ if any submodule of $M$ isomorphic to a summand of $M$ is itself a summand. $M$ is called continous if $M$ is $C S$ and has $\left(C_{2}\right)$ (Mohamed and Müller, 1990). A module $M$ is said to be an ACS-module if for every element $a \in M, R a=P \oplus S$ where $P$ is projective and $S$ is singular (Nicholson and Yousif, 2001).

By Corollary 2.11 a ring $R$ is left $Z\left({ }_{R} R\right)$-semiregular if and only if every projective module $M$ is $Z(M)$-semiregular.

If $R$ is left $Z\left({ }_{R} R\right)$-semiregular, then $Z\left({ }_{R} R\right)$ satisfies $\left(R_{1}\right)$ since $Z\left({ }_{R} R\right) \leq J(R)$. Furthermore

Proposition 3.1. Let $M$ be a projective module with $\delta(M) \ll_{\delta} M$. Then the following conditions are equivalent.

(1) $Z(M)$ satisfies $\left(R_{1}\right)$.

(2) $Z(M)$ satisfies $\left(R_{1}\right)$.

(3) $Z(M) \leq \delta(M)$.

(4) $Z(M) \leq \operatorname{Rad}(M)$.

Proof. $\quad(1) \Rightarrow(2)$. It is clear.

(2) $\Rightarrow(3)$. Since $Z(M) \cap M=Z(M), Z(M)=P \oplus S$ where $P$ is a summand of $M$ and $S \ll_{\delta} M$. Since $M$ is projective, $P=0$. Hence $Z(M) \ll_{\delta} M$.

(3) $\Rightarrow(4)$. Since $Z(M) \ll_{\delta} M$ and $Z(M)$ is singular, $Z(M) \ll M$.

$(4) \Rightarrow(1)$. It is clear.

It is proved in Nicholson and Yousif (2001, Theorem 2.4) that a ring $R$ is a left $Z\left({ }_{R} R\right)$-semiregular if and only if $R$ is semiregular and $J(R)=Z\left({ }_{R} R\right)$ if and only if $R$ is a left ACS-ring with $\left(C_{2}\right)$. Now we give the module theoretic version of this result.

Theorem 3.2. Let $M$ be a finitely generated projective module. Then the following conditions are equivalent.

(1) $M$ is $Z(M)$-semiregular.

(2) $M$ is semiregular and $Z(M)=\operatorname{Rad}(M)$. 
(3) $M$ is an ACS-module and every finitely generated (cyclic) projective submodule of $M$ is a summand.

(4) $M$ is an ACS-module and $M$ has $\left(C_{2}\right)$.

Proof. $\quad(1) \Rightarrow(2)$. If $M$ is $Z(M)$-semiregular, then $\operatorname{Rad}(M) \leq Z(M)$. For the converse, let $x \in Z(M)$. To show that $x \in \operatorname{Rad}(M)$, let $L \leq M$ be such that $M=R x+L$. Then $M / R x \cong L /(R x \cap L)$ is finitely generated. Let $T$ be a finitely generated submodule of $M$ such that $L /(R x \cap L)=[T+(R x \cap L)] /(R x \cap L)$. Then $M=R x+L=R x+T$. By Theorem 2.3, $T$ has a decomposition $T=P \oplus S$ where $P$ is a projective summand of $M$ and $S$ is singular. Then $R x+S \leq Z(M)$. $M=R x+T=R x+P+S$ and then $M / P$ is singular. Since $M$ is projective, $P \leq_{e} M$ (Nicholson and Yousif, 2001, Lemma 2.1). But this implies that $P=M$, because $P \leq^{\oplus} M$. Hence $M=T=L$. So $R x \ll M$.

$(2) \Rightarrow(3) \Rightarrow(4)$. They are clear.

$(4) \Rightarrow(1)$. Since $M$ is finitely generated projective, it is a summand of a finitely generated free module $F$. Let $A$ be such that $F=M \oplus A$ and $\left\{f_{i}\right\}_{i=1}^{n}$ be a basis of $F$. Write $f_{i}=m_{i}+a_{i}$ where $m_{i} \in M, a_{i} \in A$ for all $i=1, \ldots, n$. Let $x \in M$. By hypothesis, $R x=P \oplus S$ where $P$ is projective and $S$ is singular. It is enough to show that $P$ is a summand of $M$. We have an epimorphism $M \rightarrow R x$ defined by $m=r_{1} f_{1}+\cdots+$ $r_{n} f_{n}=r_{1} m_{1}+\cdots+r_{n} m_{n} \mapsto\left(r_{1}+\cdots+r_{n}\right) x, m \in M, r_{i} \in R, 1 \leq i \leq n$. Hence, we have an epimorphism from $M$ to $P$. This implies that $P$ is isomorphic to a summand of $M$. By $\left(C_{2}\right), P$ is a summand of $M$.

It is well known that if $R$ is left continuous then $R$ is semiregular and $Z\left({ }_{R} R\right)=J(R)$. By using Theorem 3.2, we prove the next result.

Theorem 3.3. Let $M$ be a finitely generated projective module. If $M$ is continuous, then $M$ is semiregular and $Z(M)=\operatorname{Rad}(M)$.

Proof. It is enough to show that $M$ is an ACS-module by Theorem 3.2. Let $x \in M$. Then there exists an epimorphism $f: M \rightarrow R x$ by the proof of $(4) \Rightarrow(1)$ of Theorem 3.2. Since $M$ is $C S$, there exists a summand $L$ of $M$ such that $\operatorname{Ker}(f)$ is essential in $L$. Let $K$ be a submodule such that $M=L \oplus K$. Then we have isomorphisms $\alpha: R x \rightarrow M / \operatorname{Ker}(f)$ and $\beta: M / L \rightarrow K$. Let $\pi$ denote the epimorphism from $M / \operatorname{Ker}(f)$ to $M / L$. Then $g:=\alpha \pi \beta: R x \rightarrow K$ is an epimorphism. Since $K$ is projective, $g$ splits. There exists a homomorphism $h: K \rightarrow R x$ such that $R x=$ $\operatorname{Im} h \oplus \operatorname{Ker}(g) \cdot \operatorname{Rx} / \operatorname{Ker}(g) \cong K \cong \operatorname{Im} h$ is projective and $\operatorname{Ker}(g)=\alpha^{-1}(L / \operatorname{Ker}(f)) \cong$ $L / \operatorname{Ker}(f)$ is singular. Hence $R x$ is a direct sum of a projective module and a singular module.

It is well known that any finite direct sum of modules having $\left(C_{2}\right)$ need not have $\left(C_{2}\right)$. By Theorems 3.2 and 2.10, we have the following corollary.

Corollary 3.4. Let $M$ be a finitely generated projective module. If $M$ is $Z(M)$ semiregular, then $M^{(n)}$ has $\left(C_{2}\right)$ for every $n \geq 1$. 
The following corollary is a generalization of Yousif (1997, Proposition 1.21) and Nicholson and Yousif (2001, Corollary 2.7).

Corollary 3.5. Let $M$ be a finitely generated projective module. Then

(1) $M$ is continuous if and only if $M$ is $Z(M)$-semiregular and $M$ is CS.

(2) The following are equivalent.

(a) $M$ is quasi-injective.

(b) $M$ is $Z(M)$-semiregular and $M \oplus M$ is $C S$.

(c) $M$ has $\left(C_{2}\right)$ and $M \oplus M$ is $C S$.

(d) $M$ is continuous and $M \oplus M$ is $C S$.

Proof. (1) is clear by Theorems 3.2 and 3.3. (2) (a) $\Rightarrow$ (c). By Mohamed and Müller (1990, Proposition 1.18). (c) $\Rightarrow$ (b). If $M \oplus M$ is $C S$, then $M$ is $C S$. By Theorem 3.3, $M$ is $Z(M)$-semiregular. (b) $\Rightarrow$ (a). By Corollary 3.4, $M \oplus M$ has $\left(C_{2}\right)$. Then $M \oplus M$ is continuous. By Mohamed and Müller (1990, Theorem 3.16), $M$ is quasi-injective. (c) $\Leftrightarrow(\mathrm{d})$ is clear.

\section{4. $\delta(M)$ AND $\operatorname{Soc}(M)$}

In this section, we investigate $\delta(M)$-semiregular and $\operatorname{Soc}(M)$-semiregular modules. If a module $M$ is semiregular, then it is $\delta(M)$-semiregular since $\operatorname{Rad}(M) \leq \delta(M)$. The converse is true for finitely generated modules $M$ with $\operatorname{Soc}(M)=\operatorname{Rad}(M)$ by Lemma 2.4. If $M$ is a projective module then $\delta(M)$ is equal to the intersection of all essential maximal submodules of $M$ (Zhou, 2000, Lemma 1.9), and hence $\operatorname{Soc}(M) \leq \delta(M)$. So any projective $\operatorname{Soc}(M)$-semiregular module $M$ is $\delta(M)$-semiregular. Also we will prove in Corollary 4.6 that projective $\operatorname{Soc}(M)$ semiregular modules are semiregular. Then we have the following implications for a projective module $M$.

$M$ is $\operatorname{Soc}(M)$-semiregular $\Longrightarrow M$ is semiregular $\Longrightarrow M$ is $\delta(M)$-semiregular.

By Theorem 3.2, for a finitely generated projective module $M$, we have that

$M$ is $Z(M)$-semiregular $\Longrightarrow M$ is semiregular $\Longrightarrow M$ is $\delta(M)$-semiregular.

For the converse implications we give the examples at the end of the paper.

Remark 4.1. (1) Zhou (2000, Theorem 3.5), proved that $R$ is left $\delta\left({ }_{R} R\right)$-semiregular if and only if $R / \delta\left({ }_{R} R\right)$ is regular and idempotents can be lifted modulo $\delta\left({ }_{R} R\right)$. Indeed this result follows from Theorem 2.12 because $\delta\left({ }_{R} R\right)$ satisfies $\left(S_{2}\right)$.

(2) Also $\operatorname{Soc}\left({ }_{R} R\right)$ satisfies $\left(S_{2}\right)$, since $\operatorname{Soc}\left({ }_{R} R\right) \leq \delta\left({ }_{R} R\right)$. Hence $R$ is left $\operatorname{Soc}\left({ }_{R} R\right)$ semiregular if and only if $R / \operatorname{Soc}\left({ }_{R} R\right)$ is regular and idempotents can be lifted modulo $\operatorname{Soc}\left({ }_{R} R\right)$. Baccella proved that for any ring $R$, idempotents can be lifted modulo $\operatorname{Soc}\left({ }_{R} R\right)$ (see Yousif and Zhou, 2002, Lemma 1.2). Thus $R$ is left $\operatorname{Soc}\left({ }_{R} R\right)$-semiregular if and only if $R / \operatorname{Soc}\left({ }_{R} R\right)$ is regular (see Yousif and Zhou, 2002, Theorem 1.6). 
By Corollary 2.11, a ring $R$ is left $\operatorname{Soc}\left({ }_{R} R\right)\left(\delta\left({ }_{R} R\right)\right)$-semiregular if and only if every projective module $M$ is $\operatorname{Soc}(M)(\delta(M))$-semiregular.

The next result is a structure theorem for countably generated $\delta(\cdot)$-semiregular modules.

Theorem 4.2. Let $M$ be a countably generated $\delta(M)$-semiregular module. If $\delta(M)$ is $\delta$-small in $M$ then $M$ is isomorphic to a direct sum of projective cyclic submodules.

Proof. Let $x_{1}, x_{2}, \ldots$ be a generating set for $M$. There is a decomposition $M=P_{1} \oplus Q_{1}$ such that $P_{1} \leq R x_{1}$ is projective and $K_{1}=Q_{1} \cap R x_{1}$ is $\delta$-small in $M$. As a summand of $R x_{1}$, the module $P_{1}$ is cyclic. Now we use induction. Assume, for a positive integer $n, M$ has a decomposition $M=\left(\sum_{i=1}^{n} P_{i}\right) \oplus Q_{n}$ such that $\sum_{i=1}^{n} R x_{i} \subset\left(\bigoplus_{i=1}^{n} P_{i}\right)+K_{n}$, where $K_{n}$ is $\delta$-small in $M$.

Since $Q_{n}$ is a summand of $M$ and $\delta\left(Q_{n}\right)=Q_{n} \cap \delta(M), Q_{n}$ is $\delta\left(Q_{n}\right)$-semiregular. Then there is a decomposition $Q_{n}=P_{n+1} \oplus Q_{n+1}$ such that $P_{n+1} \leq R x_{n+1}$ is projective and $T=Q_{n+1} \cap R x_{n+1}$ is $\delta$-small in $Q_{n}$. Hence $M=\left(\sum_{i=1}^{n+1} P_{i}\right) \oplus Q_{n+1}$ and $\sum_{i=1}^{n+1} R x_{i} \subset\left(\bigoplus_{i=1}^{n+1} P_{i}\right)+K_{n+1}$, where $K_{n+1}=K_{n}+T$ is $\delta$-small in $M$. Since $K=\sum_{i \in \mathbb{N}} K_{i} \leq \delta(M)$, it is $\delta$-small in $M$ and by Lemma 2.4 there exists a projective semisimple submodule $P$ of $K$ such that $M=\sum_{i \in \mathbb{N}} R x_{i}=\left(\bigoplus_{i \in \mathbb{N}} P_{i}\right)+K=$ $\left(\bigoplus_{i \in \mathbb{N}} P_{i}\right) \oplus P$. The proof is completed.

Corollary 4.3. Any finitely generated $\delta(M)$-semiregular module $M$ is projective and $Z(M) \leq \operatorname{Rad}(M)$.

Proof. By Theorem 2.3 and Proposition 3.1, $Z(M) \leq \operatorname{Rad}(M)$.

Since every projective module is a direct sum of countably generated submodules we have,

Corollary 4.4. Any projective $\delta(M)$-semiregular module $M$ with $\delta(M) \ll_{\delta} M$ is isomorphic to a direct sum of cyclic submodules.

We have mentioned that if $M$ is a projective $\operatorname{Soc}(M)$-semiregular module then $M$ is $\delta(M)$-semiregular. These modules are also semiregular and hence this result is a generalization of Yousif and Zhou (2002, Corollary 1.7(2)).

Theorem 4.5. If $M$ is a $\operatorname{Soc}(M)$-semiregular module and $Z(M) \leq \operatorname{Rad}(M)$, then $M$ is semiregular.

Proof. Let $x \in M$ and $M=A \oplus B$ where $A \leq R x$ is projective and $R x \cap B \leq$ $\operatorname{Soc}(M)$. Then $R x=A \oplus(R x \cap B)$. Assume that $R x \cap B$ has a simple submodule $S_{1}$ such that $S_{1} \nsubseteq \operatorname{Rad}(M)$, if not every simple submodule of $\operatorname{Rx} \cap B$ is in $\operatorname{Rad}(M)$ and hence this completes the proof. Then $S_{1}$ is a summand of $M$, and hence summand of $B$. Let $L_{1}$ be such that $B=S_{1} \oplus L_{1}$. Then $R x \cap B=S_{1} \oplus\left(R x \cap L_{1}\right)$ and $M=A \oplus S_{1} \oplus L_{1}$. This implies that $R x=\left(A \oplus S_{1}\right) \oplus\left(R x \cap L_{1}\right)$. 
Similarly since $R x \cap L_{1}$ is semisimple assume that $R x \cap L_{1}$ has a simple submodule $S_{2}$ such that $S_{2} \nsubseteq \mathbb{R a d}(M)$, if not again the proof is completed. Since $S_{2}$ is a summand of $M$, there exists a submodule $L_{2}$ such that $L_{1}=S_{2} \oplus L_{2}$. It follows that $R x \cap L_{1}=S_{2} \oplus\left(R x \cap L_{2}\right)$ and $M=A \oplus S_{1} \oplus S_{2} \oplus L_{2}$. Then $R x=\left(A \oplus S_{1} \oplus S_{2}\right) \oplus$ $\left(L_{2} \cap R x\right)$. This process produces a strictly descending chain $B \cap R x \supset L_{1} \cap R x \supset$ $L_{2} \cap R x \supset \cdots$. Since $B \cap R x$ is semisimple and finitely generated, it is Artinian. Hence this process must stop, so that $L_{n} \cap R x \leq \operatorname{Rad}(M)$ for some positive integer $n$. Hence $R x=\left(A \oplus S_{1} \oplus \cdots \oplus S_{n}\right) \oplus\left(L_{n} \cap R x\right)$. So $M$ is semiregular.

Corollary 4.6. Any projective $\operatorname{Soc}(M)$-semiregular module $M$ is semiregular.

Proof. Since $Z(M) \leq \operatorname{Soc}(M)$, let $S$ be a singular simple submodule of $M$. If $S \nsubseteq \operatorname{Rad}(M)$, then $S$ is a summand of $M$. This implies that $S=0$. Hence $Z(M) \leq$ $\operatorname{Rad}(M)$. By Theorem 4.5, $M$ is semiregular.

Corollary 4.7. Let $M$ be a finitely generated $\operatorname{Soc}(M)$-semiregular module. Then $M$ is projective if and only if $Z(M) \leq \operatorname{Rad}(M)$.

Proof. It is clear by Theorem 4.5 and Corollary 4.3 .

Hence if $M$ is a projective $\operatorname{Soc}(M)$-semiregular module then

$$
Z(M) \leq \operatorname{Rad}(M) \leq \operatorname{Soc}(M) \leq \delta(M)
$$

If $R$ is a left $\operatorname{Soc}\left({ }_{R} R\right)$-semiregular ring, then $\delta\left({ }_{R} R\right)=\operatorname{Soc}\left({ }_{R} R\right)$. For, $\delta\left({ }_{R} R\right) / \operatorname{Soc}\left({ }_{R} R\right)=J\left(R / \operatorname{Soc}\left({ }_{R} R\right)\right)=0$ (Zhou, 2000, Corollary 1.7). Also $J(R)^{2}=0$ because $J(R) \operatorname{Soc}\left({ }_{R} R\right)=0$. But this does not necessarily hold if $R$ is semiregular. For example there exists a local ring $R$ such that $J(R)$ is not nilpotent (see Zhou, 2000, Example 4.4 for the existence of such a ring). Then $R$ is semiregular but $J(R)^{2} \neq 0$.

Proposition 4.8. If a module $M$ is Soc(M)-semiregular, then $M$ is an ACS-module.

Proof. Let $a \in M$. Then $R a=A \oplus B$ where $A$ is a projective summand of $M$ and $B \leq \operatorname{Soc}(M)$. Let $B=B_{1} \oplus B_{2}$ where $B_{1}$ is a direct sum of projective simples and $B_{2}$ is a direct sum of singular simples. Then $R a=A \oplus B_{1} \oplus B_{2}$ where $A \oplus B_{1}$ is projective and $B_{2}$ is singular.

Next we consider the Noetherian $\operatorname{Soc}(M)$-semiregular modules.

Theorem 4.9. Any Noetherian Soc(M)-semiregular module $M$ is Artinian.

Proof. If $M$ is Noetherian $\operatorname{Soc}(M)$-semiregular, $M / \operatorname{Soc}(M)$ is semisimple by Theorem 2.12. Since $M$ is Noetherian, $M / \operatorname{Soc}(M)$ is Artinian and so $M$ is Artinian. 
Corollary 4.10. The following conditions are equivalent for a ring $R$.

(1) $R$ is a left Artinian ring with $J(R)^{2}=0$.

(2) $R$ is a left Noetherian left $\operatorname{Soc}\left({ }_{R} R\right)$-semiregular ring.

Proof. $\quad(2) \Rightarrow(1)$. It is clear.

$(1) \Rightarrow(2)$. Since the left annihilator of $J(R)$ is $\operatorname{Soc}\left({ }_{R} R\right), J(R) \leq \operatorname{Soc}\left({ }_{R} R\right)$. Left Artinian rings are semiregular. Hence $R$ is left $\operatorname{Soc}\left({ }_{R} R\right)$-semiregular.

From now on, we deal with $\operatorname{Soc}(M)$-semiregular modules $M$ such that $M$ has $\left(C_{2}\right)$ or is min- $C S$ or $C S$.

Proposition 4.11. Let $M$ be a finitely generated projective module. Then the following conditions are equivalent.

(1) $M$ is $\operatorname{Soc}(M)$-semiregular with $\left(C_{2}\right)$.

(2) $M$ is $\operatorname{Soc}(M)$-semiregular and $Z(M)=\operatorname{Rad}(M)$.

(3) $M$ is $\operatorname{Soc}(M)$-semiregular and every simple projective submodule of $M$ is a summand.

(4) $M$ is $Z(M)$-semiregular and $Z(M) \leq \operatorname{Soc}(M)$.

Proof. (4) $\Rightarrow(1)$ and (4) $\Rightarrow(2)$ are clear. (1) $\Rightarrow(4)$ is by Theorem 3.2 and Proposition 4.8

(2) $\Rightarrow(3)$. Let $S$ be a projective simple submodule of $M$. Then $S \nsubseteq \operatorname{Rad}(M)$ and hence $S$ is a summand of $M$.

(3) $\Rightarrow(4)$. Let $x \in M$. Then $M$ has a decompositon $M=A \oplus B$ such that $A$ is a projective submodule of $R x$ and $B \cap R x \leq \operatorname{Soc}(M)$. Then $R x=A \oplus(B \cap R x)$. Let $B \cap R x=S_{1} \oplus S_{2}$ where $S_{1}$ is a finite direct sum of projective simples and $S_{2}$ is a finite direct sum of singular simples. Then $S_{1}$ is a summand of $B$ by the similar proof of Mohamed and Müller (1990, Proposition 2.2). Hence $A \oplus S_{1}$ is a summand of $M$. This implies that $M$ is $Z(M)$-semiregular.

By Theorems 2.10 and 3.2, if $M$ is a finitely generated projective $\operatorname{Soc}(M)$-semiregular module with $\left(C_{2}\right)$, then $M^{(n)}$ is $\operatorname{Soc}\left(M^{(n)}\right)$-semiregular and has $\left(C_{2}\right)$ for every $n \geq 1$.

For the following corollary see also Yousif and Zhou (2002, Theorem 2.11).

Corollary 4.12. The following conditions are equivalent for a ring $R$.

(1) $R$ is left $\operatorname{Soc}\left({ }_{R} R\right)$-semiregular, $R / \operatorname{Soc}\left({ }_{R} R\right)$ is Noetherian and any projective semisimple left ideal is a summand.

(2) $R$ is semiprimary and $J(R)=Z\left({ }_{R} R\right) \leq \operatorname{Soc}\left({ }_{R} R\right)$.

Proof. $\quad(1) \Rightarrow(2)$. By Corollary 2.13 and the hypothesis, $R$ is semiperfect. Since $J(R)^{2}=0, R$ is semiprimary. By Proposition 4.11, $J(R)=Z\left({ }_{R} R\right)$. 
$(2) \Rightarrow(1)$. Since $R$ is semiprimary, it is semiregular and $\mathrm{R} / \mathrm{J}(\mathrm{R})$ is semisimple Artinian. Since $J(R) \leq \operatorname{Soc}\left({ }_{R} R\right), R$ is left $\operatorname{Soc}\left({ }_{R} R\right)$-semiregular and $R / \operatorname{Soc}\left({ }_{R} R\right)$ is Noetherian. Since $J(R)=Z\left({ }_{R} R\right)$, any projective semisimple left ideal is a summand.

A module $M$ is called a min-CS module if every simple submodule of $M$ is essential in a summand of $M$. A ring $R$ is called left min- $C S$ ring if ${ }_{R} R$ is a min-CS module.

Proposition 4.13. Let $M$ be a Noetherian projective module. Then the following conditions are equivalent.

(1) $M$ is continuous and $\operatorname{Rad}(M) \leq \operatorname{Soc}(M)$.

(2) $M$ is $\operatorname{Soc}(M)$-semiregualr, min-CS with $\left(C_{2}\right)$.

Proof. $\quad(1) \Rightarrow(2)$. It is clear by Theorem 3.3.

(2) $\Rightarrow(1)$. We claim that $M$ is $C S$. Let $N$ be a submodule of $M$. Then $N$ has a decomposition $N=A \oplus S$ such that $A$ is a summand of $M$ and $S \leq \operatorname{Soc}(M)$. Since $M$ is min- $C S$ and by Mohamed and Müller (1990, Proposition 2.2), there exists a summand $C$ of $M$ such that $S \leq_{e} C$. Then $N \leq_{e} A \oplus C \leq^{\oplus} M$. Hence $M$ is $C S$.

A ring $R$ is called left Kasch if every simple left $R$-module is embedded in $R$, or equivalently, for any maximal left ideal $I$ in $R$, the right annihilator of $I$ is nonzero. By Therorem 4.9 and Yousif (1997, Theorem 1.16), we have the following corollary.

Corollary 4.14. Let $R$ be a left Noetherian ring. The following conditions are equivalent.

(1) $R$ is left continuous with $J(R) \leq \operatorname{Soc}\left({ }_{R} R\right)$.

(2) $R$ is left $\operatorname{Soc}\left({ }_{R} R\right)$-semiregular left min-CS and left $\left(C_{2}\right)$.

In this case $R$ is a left Artinian left and right Kasch ring.

If a ring $R$ is left Artinian left continuous left and right Kasch with $J(R) \leq \operatorname{Soc}\left({ }_{R} R\right), R$ need not be a QF-ring:

Example 4.15 (Björk, 1970). Given a field $F$ and an isomorphism a $\mapsto \bar{a}$ from $F \rightarrow$ $\bar{F} \subseteq F$, let $R$ be the right $F$-space on basis $\{1, \mathrm{t}\}$ with multiplication given by $t^{2}=0$ and at $=\mathrm{t} \bar{a}$ for all $\mathrm{a} \in F$. Then $R$ is a local ring and the only right ideals are $0, J(R)$ and $R$. Hence $R$ is right Artinian right continuous and left and right Kasch. It follows that $J(R)=\operatorname{Soc}\left({ }_{R} R\right)=\operatorname{Soc}\left({ }_{R} R\right)$. If $\operatorname{dim}_{\bar{F}}(F) \geq 2$, then $R$ is not left continuous (see Yousif and Zhou, 2002, Example 2.17).

Theorem 4.16. Let $M$ be a finitely generated module. Then the following conditions are equivalent.

(1) $M$ is $C S$ and $M / \operatorname{Soc}(M)$ is semisimple.

(2) $M$ is $C S$ Artinian and $\operatorname{Rad}(M) \leq \operatorname{Soc}(M)$. 
In addition if $M$ is projective, (1) and (2) are equivalent to

(3) $M$ is $C S \operatorname{Soc}(M)$-semiregular and $M / \operatorname{Soc}(M)$ is Noetherian.

Proof. $\quad(1) \Rightarrow(2)$. Since $M / \operatorname{Soc}(M)$ is semisimple, $\operatorname{Rad}(M) \leq \operatorname{Soc}(M)$. By Dung et al. (1994, 5.15 and 18.7), $M$ is Artinian.

$(2) \Rightarrow(1)$. Since $M$ is Artinian, $M / \operatorname{Rad}(M)$ is semisimple.

$(2) \Rightarrow(3)$. Since $M$ is Artinian and projective, $M$ is semiregular (Wisbauer, $1991,41.15)$ and $M / \operatorname{Rad}(M)$ is semisimple. Then $M$ is $\operatorname{Soc}(M)$-semiregular and $M / \operatorname{Soc}(M)$ is semisimple.

(3) $\Rightarrow(1)$. By Theorem 2.12, $M / \operatorname{Soc}(M)$ is semisimple.

Corollary 4.17. The following conditions are equivalent for a ring $R$.

(1) $R$ is left CS left Artinian with $J(R)^{2}=0$.

(2) $R$ is left CS left $\operatorname{Soc}\left({ }_{R} R\right)$-semiregular and $R / \operatorname{Soc}\left({ }_{R} R\right)$ is left Noetherian.

Theorem 4.18. Let $M$ be finitely generated projective module. The following conditions are equivalent.

(1) $M$ is Artinian quasi-injective and $\operatorname{Rad}(M) \leq \operatorname{Soc}(M)$.

(2) $M$ has $\left(C_{2}\right), M \oplus M$ is $C S$ and $M / \operatorname{Soc}(M)$ is semisimple.

(3) $M$ is Noetherian $\operatorname{Soc}(M)$-semiregular with $\left(C_{2}\right)$ and $M \oplus M$ is min-CS.

Proof. (1) $\Rightarrow(2)$. Since $M$ is quasi-injective, $M \oplus M$ is $C S$ by Mohamed and Müller (1990, Proposition 1.18).

$(2) \Rightarrow(3)$. Since $M$ is $C S$ and $M / \operatorname{Soc}(M)$ is Artinian and Noetherian, $M$ is Artinian and Noetherian by Dung et al. (1994, 5.15 and 18.17). Since $M$ is Artinian and projective, it is semiregular (Wisbauer, 1991, 41.15). Since $\operatorname{Rad}(M) \leq \operatorname{Soc}(M), M$ is $\operatorname{Soc}(M)$-semiregular.

(3) $\Rightarrow(1)$. Then $M \oplus M$ is $\operatorname{Soc}(M \oplus M)$-semiregular and by Proposition 4.11 and 4.13, $Z(M \oplus M)=\operatorname{Rad}(M \oplus M)$ and $M \oplus M$ is continuous. Hence $M$ is quasiinjective (Mohamed and Müller, 1990, Theorem 3.16).

Note that a left self-injective (resp. right and left continuous) ring $R$ such that $R / \operatorname{Soc}\left({ }_{R} R\right)$ is left Noetherian is QF (Ara and Park, 1991). But there exists a Noetherian projective self-injective module which is not Artinian (see Dung et al., 1994, Example in p. 87). Hence in the above theorem it is not enough for $M$ to be Artinian to assume that $M / \operatorname{Soc}(M)$ is Noetherian.

Corollary 4.19. The following conditions are equivalent for a ring $R$.

(1) $R$ is a $Q F$-ring with $J(R)^{2}=0$.

(2) ${ }_{R} R$ has $\left(C_{2}\right),{ }_{R}(R \oplus R)$ is $C S$ and $R / \operatorname{Soc}\left({ }_{R} R\right)$ is semisimple Artinian.

(3) $R$ is left $\operatorname{Soc}\left({ }_{R} R\right)$-semiregular, left Noetherian with left $\left(C_{2}\right)$ and $R \oplus R$ is left min-CS. 
Now we give the examples. First example shows that there is a projective module $M$ which is $\delta(M)$-semiregular but not semiregular hence not $\operatorname{Soc}(M)$-semiregular (see Nicholson, 1976, Example 2.15).

Example 4.20. Let $F$ be a field, $I=\left[\begin{array}{ll}F & F \\ 0 & F\end{array}\right]$ and

$M=R=\left\{\left(x_{1}, \ldots, x_{n}, x, x, \ldots\right): n \in \mathbb{N}, x_{i} \in M_{2}(F), x \in I\right\}$.

With component-wise operations, $R$ is a ring.

$$
\begin{aligned}
& \delta\left({ }_{R} R\right)=\left\{\left(x_{1} \ldots, x_{n}, x, x, \ldots\right): n \in \mathbb{N}, x_{i} \in M_{2}(F), x \in J\right\} \quad \text { where } \quad J=\left[\begin{array}{ll}
0 & F \\
0 & 0
\end{array}\right] . \\
& \operatorname{Soc}\left({ }_{R} R\right)=\left\{\left(x_{1}, \ldots, x_{n}, 0,0, \ldots\right): n \in \mathbb{N}, x_{i} \in M_{2}(F)\right\}
\end{aligned}
$$

Thus,

$$
R / \operatorname{Soc}\left({ }_{R} R\right) \cong\left[\begin{array}{cc}
F & F \\
0 & F
\end{array}\right]
$$

and so $R$ is not left $\operatorname{Soc}\left({ }_{R} R\right)$-semiregular. Also by Example 2.15 in Nicholson (1976) $R$ is not semiregular, but $\delta\left({ }_{R} R\right)$-semiregular by Example 4.3 in Zhou (2000).

If $M$ is finitely generated projective $Z(M)$-semiregular, then $M$ need not be $\operatorname{Soc}(M)$-semiregular. Hence there is a module $M$ which is semiregular but not $\operatorname{Soc}(M)$-semiregular (see also Yousif and Zhou, 2002, Example 1.8).

Example 4.21. Let $M=R=\mathbb{Z}_{8}$. Then $R$ is a self-injective ring, $J(R)=Z(R)=2 R$ and $\operatorname{Soc}(R)=4 R$. Hence $R$ is a $Z(R)$-semiregular ring by Nicholson and Yousif (2001) but not $\operatorname{Soc}(R)$-semiregular since $J(R)$-semiregular is not contained in $\operatorname{Soc}(R)$.

If $M$ is $\operatorname{Soc}(M)$-semiregular then $M$ need not be $Z(M)$-semiregular. The ring of $2 \times 2$ upper triangular matrices over a field is the example of such a module, see Yousif and Zhou, 2002, Example 1.8).

\section{ACKNOWLEDGMENTS}

The authors would like to give their special thanks to the referee for his/her valuable suggestions that have improved the quality of the presentation of this paper. Also it is a pleasure to thank Prof. D. V. Huynh (Ohio University) for his helpful comments.

\section{REFERENCES}

Anderson, F. W., Fuller, K. R. (1974). Rings and Categories of Modules. New-York: Spring-Verlag.

Ara, P., Park, J. K. (1991). On continuous semiprimary rings. Comm. Alg. 19(7):1945-1957. 
Azumaya, G. (1991). F-semiperfect modules. J. Alg. 136:73-85.

Björk, J. E. (1970). Rings satisfying certain chain conditions. J. Reine Angew. Math. 245:63-73.

Dung, N. V., Huynh, D. V., Smith, P. F., Wisbauer, R. (1994). Extending Modules. London: Pitman.

Mares, E. (1963). Semiperfect modules. Math Zeitschr. 82:347-360.

Mohamed, S. H., Muller, B. J. (1990). Continuous and Discrete Modules. London Mathematical Society, Lecture Notes Series 147. Cambridge: Cambridge Univ. Press.

Nicholson, W. K. (1976). Semiregular modules and rings. Canad. Math. J. 28(5):1105-1120.

Nicholson, W. K., Yousif, M. F. (2001). Weakly continuous and C2 conditions. Comm. Alg. 29(6):2429-2446.

Wisbauer, R. (1991). Foundations of Modules and Ring Theory. Gordon and Breach.

Xue, W. (1995). Semiregular modules and F-semiperfect modules. Comm. Alg. 23(3):1035-1046.

Yousif, M. F. (1997). On continuous rings. J. Alg. 191:495-509.

Yousif, M. F., Zhou, Y. (2002). Semiregular semiperfect and perfect rings relative to an ideal. Rocky Mountain J. Math. 32(4):1651-1671.

Zelmanowitz, J. (1973). Regular modules. Trans. Amer. Math. Soc. 163:341-355.

Zhou, Y. (2000). Generalizations of perfect, semiperfect and semiregular rings. Alg. Coll. 7(3):305-318.

Received May 2003

Revised August 2003 